\title{
Pharmacogenetics of asthma
}

\author{
I.P. Hall
}

Clinicians are always struck by the marked disparity in treatment response between individuals with a given disease of a particular severity. There are many explanations for variability in treatment response and these can be split up into defined categories (table 1). This perspective deals with the genetic factors which may potentially play a role in determining treatment response in patients with asthma.

The study of pharmacogenetics is in its infancy. However, the idea that genetic factors may be important in determining treatment response to a given drug is not new. The best examples of pharmacogenetic factors influencing drug treatment response relate to drug metabolizing enzymes. For example, individuals carrying particular cytochrome P4502D6 alleles show altered metabolism of several clinically important drugs including nortryptylline and the clinical effectiveness (and incidence of side effects) on treatment is largely determined by genotype. Other examples include the role of P4502C9 alleles in warfarin metabolism and cytochrome P4502A6 alleles in nicotine dependence [1-4]. The magnitude of effect of these genetic variants is considerable. Indeed a case could probably be made on pharmacoeconomic grounds for routine screening of individuals before commencing isoniazid therapy (especially in racial groups with a high prevalence of the slow metabolizer status). Interestingly, a deoxyribonucleic acid (DNA) chip is already marketed in the USA and Scandinavia for genotyping of cytochrome P450 alleles.

Few agents used in the treatment of asthma have critical metabolic pathways for which genetic variants control drug levels. However, attention has recently focused on the possibility that the response to a given agent at the cell level may be determined by genetic variants of the target protein. Polymorphism within genes within the human genome is common. In general $\sim 1$ in 1,000 base pairs within coding regions are polymorphic, although because of redundancy in the amino acid coding system not all single nucleotide polymorphisms (SNPs) result in an amino acid change in the relevant protein. The rate of polymorphic variation also varies at different loci, with some genes having much higher levels of polymorphism. A recent screen of candidate genes for hypertension suggested the majority contained at least one SNP [5]. A comprehensive screen of genes likely to be important in determining asthma treatment response has not been performed, although the author's group and others have been systematically looking at the genes coding for primary targets, searching for polymorphic variation. However, it is important to realize

Correspondence: J.P. Hall, Division of Therapeutics, C Floor South Block, University Hospital, Nottingham, NG7 2UH, UK. Fax: 44 1159422232.

that most polymorphisms are likely to have no major functional effects, and so before searching for clinical associations between a given polymorphism and a treatment end-point, it is essential to assess the potential functional effects of the polymorphism in question. A summary of the main variants in asthma treatment response genes which have been identified, to date is shown in table 2 .

For a polymorphic variant to be important in determining treatment response at the population level it must be reasonably common. A good example of a rare polymorphism which may be important, but only in occasional individuals, is the threonine-isoleucine (Thr-Ile) $164 \beta_{2}$-adrenoceptor polymorphism. In vitro functional studies in recombinant cell systems have demonstrated that the isoleucine $164 \beta_{2^{-}}$ adrenoceptor variant shows markedly reduced coupling to catechol ligands and it could be predicted that individuals homozygous for this variant (all individuals carry two copies of the $\beta_{2}$-adrenoceptor gene which is situated on chromosome $5 \mathrm{q}$ ) would show a markedly reduced response to many $\beta_{2}$-agonists [15]. However, the allelic frequency of this polymorphism in the Caucasian population is only $\sim 3 \%$ and to date the author's group have not identified a homozygous individual. In contrast the arginine-glycine (Arg-Gly) 16 polymorphism in the $\beta_{2}$-adrenoceptor is common in the general population [9]. The glycine 16 form of the receptor in vitro demonstrated increased down regulation following agonist exposure [16] and two clinical studies have suggested that individuals homozygous for the glycine 16 variant of the $\beta_{2}$-adrenoceptor show reduced treatment response following either acute or chronic exposure to $\beta_{2}$-agonists $[7,17]$. The magnitude of effect in these studies was relatively small and other studies have shown no association of this polymorphism with fatal asthma or long term deterioration on $\beta_{2}$-agonists [18]. The key question as to whether individuals homozygous for the glycine-16 variant of the $\beta_{2}$-adrenoceptor show reduced responsiveness to long acting $\beta_{2}$-agonist remains to be fully assessed. If indeed reduced responses

Table 1. - Some factors affecting treatment response

Disease specific
Disease severity
Disease subtype (e.g. aspirin-sensitive asthma)
Compliance
Individual factors
Genetic
Intercurrent illness
Other medication
Environmental exposures
Age

Disease specific

Disease severity

Compliance

Individual factors

Environmental exposures 
Table 2. - Asthma treatment target gene polymorphisms

\begin{tabular}{lcc}
\hline Gene & Polymorphism & Clinically relevant \\
\hline$\beta_{2}$-adrenoceptor & Arg-Gly 16 & Yes \\
& Gln-Glu 27 & Uncertain \\
& Thr-Ile 164 & Yes \\
& -47 C-T & ?No \\
$\mathrm{H}_{1}$ receptor & Gln 356 & No \\
$\mathrm{M}_{2}$ receptor & $+1696 \mathrm{~T}-\mathrm{A}$ & Unlikely \\
$\mathrm{M}_{3}$ receptor & Not known & Not known \\
5 -LO & Alleles 1-5 & ?Yes \\
LTD & Not known & Not known \\
GR & Asn 363-Ser & - \\
PDE IV A-D & Several & Not known \\
\hline
\end{tabular}

Arg: arginine; Gly: glycine; Gln: glutamine; Glu: glutamic acid; Thr: threonine; Ile: isoleucine; Asn: asparagine; Ser: serine; C: cytosine; T: thymidine; $\mathrm{H}_{1}$ receptor: histamine type 1 receptor; $\mathrm{M}_{2}$ receptor: muscarinic type 2 receptor; A: adenine; $M_{3}$ receptor: muscarinic type 3 receptor; 5-LO: 5-lipoxygenase; $\mathrm{LTD}_{4} \mathrm{R}$ : leukotriene $\mathrm{D}_{4}$ receptor; GR: glucocorticoid receptor; PDE IV A-D: phosphodiesterase type 4 isozyme family, forms A-D. See references [6-14].

in these individuals were found, a pharmacoeconomic evaluation would need to be performed to see whether alternative treatments appropriate for such patients at steps 3-4 of the British Thoracic Society Asthma Treatment Guidelines (e.g. high dose inhaled steroid, theophylline) [19] should be given before long acting $\beta_{2-}$ agonists and to determine whether or not the costs of genetic screening would be worthwhile to assist in decision making.

The other good example of genetic variants apparently determining treatment response is in the 5-lipoxygenase gene. The level of 5-lipoxygenase activity will in part determine the level of bronchoconstrictor leukotrienes (LTC-4, LTD-4) present in the airways [20]. Transcriptional activity of the 5-lipoxygenase gene appears in part to be dependent on transcription factor binding to a site upstream of the gene which shows genetic variation with individuals having between 3-6 tandem repeats of the transcription factor $\mathrm{Sp}-1$ binding motif GGGCGG [12]. A recent study suggested individuals who would be predicted to have low transcriptional activity of the gene because of different alleles at this site (the wild type having five copies) showed reduced clinical response to the 5lipoxygenase inhibitor ABT-761 [21]. Whether or not this polymorphism may also affect treatment response to the cysteine (Cys) leukotriene receptor antagonists montelukast and zafirlukast remains to be determined.

The author has also recently screened the muscarinic $\mathrm{M}_{2}$ receptor gene [14] and the histamine $\mathrm{H}_{1}$ receptor gene [11] looking for functionally relevant polymorphic variation. However, at least using single-stranded conformational polymorphism analysis-based methods, the author was unable to detect polymorphisms which altered the amino acid sequence of the receptor despite screening reasonably large numbers of individuals $(n=20-50)$. The $\mathrm{H}_{1}$ receptor does contain a relatively rare degenerate polymorphism at amino acid 356 (glutamine) but this is unlikely to be functionally relevant. Likewise, studies on the glucocorticoid receptor have so far failed to reveal significant variation leading to loss of function, although this is obviously an attractive candidate in view of the recognition that steroid responsiveness varies markedly between asthmatic individuals [13]. Finally, the author has recently been examining polymorphic variation in the phosphodiesterase type 4 isoform family [22]. Database searches suggest that polymorphic variants do exist in this gene family although the potential functional relevance of these polymorphisms remains to be determined. Because there are several different phosphodiesterase type 4 family members, and also splice variants of the different genes, evaluating the significance of polymorphisms in this gene family will be difficult.

In summary therefore it is clear that genes whose products are important targets for asthma treatment contain functionally relevant polymorphisms. In general the magnitude of any functional effect of these polymorphisms is smaller than for established polymorphisms in, for example, hepatic drug metabolizing pathways. Advances in chip technology have made large scale genetic screening of individuals for given polymorphisms easy to perform but no adequate studies on the cost effectiveness of this approach have been carried out to date. For such studies to proceed, an adequate assessment of the contribution of a given polymorphism to variability in response to a given drug needs to be made, and this is the challenge for asthma pharmacogenetic studies in the next few years. The possibility that pharmacogenetic factors may influence treatment response is particularly important in small phase II studies because if a small group contains by chance an excess of individuals with one particular genotype which affects treatment response it would be easy to either overestimate or underestimate the efficacy of the compound being studied. In addition the possibility that genetic variation may be important in determining the magnitude of treatment response has implications for the drug licencing process in that it is likely that regulatory bodies will need to be reassured that novel compounds coming to the market have been assessed in an appropriate population in terms of genetic variability.

Acknowledgements. This article is based upon an invited lecture at the National Asthma Campaign International Conference held in London, UK, 1999.

\section{References}

1. Oscarson M, Hidestrand M, Johansson I, IngelmanSundberg M. A combination of mutations in the CYP2D6*17 (CYP2D6Z) allele causes alterations in enzyme function. Mol Pharmacol 1997; 52: 1034-1040.

2. Dalen P, Dahl M-L, Bernal Ruiz ML, Nordin J, Bertilsson L. 10-Hydroxylation of nortriptyline in white persons with 0,1,2,3, and 13 functional CYP2D6 genes. Clin Pharmacol Ther 1998; 63: 444-452.

3. Pianeza ML, Sellers EM, Tyndale RF. Nicotine metabolism defect reduces smoking. Nature 1998; 393: 750.

4. Ingelman-Sundberg M, Oscarson M, McLellan RA. Polymorphic human cytochrome P450 enzymes: an opportunity for individualized drug treatment. Trends Pharmacol Sci 1999; 20: 342-349.

5. Halusdka MK, Fan J-B, Bentley K, et al. Patterns of single nucleotide polymorphisms in candidate genes for blood pressure homeostasis. Nature Genetics 1999; 22: 239-247.

6. Reishaus E, Innis M, MacIntyre N, Liggett SB. Mutations 
in the gene encoding for the $\beta_{2}$ adrenergic receptor in normal and asthmatic subjects. Am J Respir Cell Mol Biol 1993; 8: 334-339.

7. Tan KS, Hall IP, Dewar JC, Dow E, Lipworth BJ. $\beta_{2}$ adrenoceptor $\left(\beta_{2}\right.$-AR) polymorphism determines susceptibility to bronchodilator desensitisation in asthmatics. Lancet 1997; 4: 995-999.

8. Turki J, Pak J, Green SA, Martin RJ, Liggett SB. Genetic polymorphisms of the $\beta_{2}$ adrenergic receptor in nocturnal and nonnocturnal asthma. Evidence that Gly 16 correlates with the nocturnal phenotype. J Clin Invest 1995; 95: 1635-1641.

9. Dewar JC, Wheatley AP, Venn A, Morrison JFJ, Britton J, Hall IP. $\beta_{2}$ adrenoceptor polymorphisms are in linkage disequilibrium, but are not associated with asthma in an adult population. Clin Exp All 1998; 28: 442-448.

10. Hancox RJ, Sears MR, DR Taylor. Polymorphism of the $\beta_{2}$ adrenoceptor and the response to long-term, $B_{2}$ agonist therapy in asthma. Eur Respir J 1998; 11: 589-593.

11. Dewar JC, Hall IP. A novel degenerate polymorphism in the human histamine $\mathrm{H} 1$ receptor gene. Am J Respir Crit Care Med 1998; 157: A773.

12. In KH, Asano K, Beler D, et al. Naturally occurring mutations in the human 5-lipoxygenase gene promoter that modify transcription factor binding and reporter gene transcription. J Clin Invest 1997; 99: 1130-1137.

13. Lane SJ, Arm JP, Staynov DZ, Lee TH. Chemical mutational analysis of the human glucocorticoid receptor cDNA in glucocorticoid-resistant bronchial asthma. Am J Respir Cell Mol Biol 1994; 11: 42-44.

14. Fenech AG, Ebejer MJ, Felice AK, Ellul-Micallef R, Hall IP. Mutation screening of the human muscarinic $\mathrm{M}_{2}$ recep- tor gene in Maltese asthmatic patients. Br J Pharmacol 1999; 128: 121P.

15. Green SA, Cole G, Jacinto M, Innis M, Liggett SB. A polymorphism of the human $\beta_{2}$-receptor within the fourth transmembrane domain alters ligand binding and functional properties of the receptor. J Biol Chem 1993; 268: 23116-23121

16. Green SA, Turki J, Innis M, Liggett SB. Amino-terminal polymorphisms of the human $\beta_{2}$-adrenergic receptor impart distinct agonist-promoted regulatory properties. Biochem (USA) 1994; 33: 9414-9419.

17. Martinez FD, Graves PD, Baldini M, Solomon S, Erickson R. Association between genetic polymorphisms of the $\beta_{2}$ adrenoceptor and response to albuterol in children with and without a history of wheezing. J Clin Invest 1997; 100: 3184-3188.

18. Weir TD, Mallek N, Sandford AJ, et al. $\beta_{2}$-adrenergic receptor haplotypes in mild, moderate and fatal/near fatal asthma. Am J Respir Crit Care Med 1998; 158: 787-791.

19. The British Guidelines for Asthma Management: 1995 Review and Position Statement. Thorax 1997; 52 (Suppl. 1): $\mathrm{S} 1-\mathrm{S} 21$.

20. Drazen JM, Israel E, Obyrne PM. Treatment of asthma with drugs modifying the leukotriene pathway. $N$ Engl $J$ Med 1999; 340: 197-206.

21. Drazen JM, Yandava CN, Dube L, et al. Phamacogenetic association between ALOX5 promoter genotype and the response to anti-asthma treatment. Nature Genetics 1999; 22: $168-170$.

22. Beavo JA. Cyclic nucleotide phosphodiesterases: functional implications of multiple isoforrns. Physiol Rev 1995; 75: 725-748. 